\title{
Annotate-it: a Swiss-knife approach to annotation, analysis and interpretation of single nucleotide variation in human disease
}

\author{
Alejandro Sifrim ${ }^{1,2^{*}}$, Jeroen KJ Van Houdt ${ }^{3}$, Leon-Charles Tranchevent ${ }^{1,2}$, Beata Nowakowska ${ }^{3}$, Ryo Sakai ${ }^{1,2}$, \\ Georgios A Pavlopoulos ${ }^{1,2}$, Koen Devriendt ${ }^{3}$, Joris R Vermeesch ${ }^{3}$, Yves Moreau ${ }^{1,2}$ and Jan Aerts ${ }^{1,2}$
}

\begin{abstract}
The increasing size and complexity of exome/genome sequencing data requires new tools for clinical geneticists to discover disease-causing variants. Bottlenecks in identifying the causative variation include poor cross-sample querying, constantly changing functional annotation and not considering existing knowledge concerning the phenotype. We describe a methodology that facilitates exploration of patient sequencing data towards identification of causal variants under different genetic hypotheses. Annotate-it facilitates handling, analysis and interpretation of high-throughput single nucleotide variant data. We demonstrate our strategy using three case studies. Annotate-it is freely available and test data are accessible to all users at http://www.annotate-it.org.
\end{abstract}

\section{Background \\ Context}

With the advent of massively parallel high-throughput sequencing technologies and the increasing availability of reference genomes, new opportunities emerge for discovering genome-wide variation across individuals and populations, at both the large-scale level (deletions, duplications, and rearrangements) and the base-pair level (single nucleotide variants and small indels and repeats). As more and more sequence data are produced, accurate assessment of the frequency of variants in specific subpopulations (patients versus controls or any phenotypically different populations) is vital to the interpretation of how these variants segregate across populations. International projects, such as the 1000 Genomes Project [1] and the Hapmap Project [2], have been set up to assess the genetic variation across large groups of 'normal' human individuals. Full-genome [3], whole-exome [4-6] or targeted gene panel $[7,8]$ sequencing studies of individuals struck by Mendelian disorders can aid in identifying the genetic cause for these diseases; for example, by leveraging publicly available data, under the assumption that

\footnotetext{
* Correspondence: alejandro.sifrim@esat.kuleuven.be

${ }^{1} \mathrm{KU}$ Leuven, Department of Electrical Engineering-ESAT, SCD-SISTA,

Kasteelpark Arenberg 10, B-3001, Leuven, Belgium

Full list of author information is available at the end of the article
}

these rare variants do not occur in the normal population. Furthermore, trio sequencing of an affected child and of his or her parents can identify de novo variants in sporadic cases of genetic disease $[9,10]$.

Because of the large size and complexity of nextgeneration sequencing data sets, new computational and statistical methods for analyzing and interpreting the data are required to accurately find the variation of biological interest. To transform raw sequencing data into variation data, we need to undertake the following steps: (1) sequence alignment, (2) variant calling, (3) variant annotation, and (4) variant interpretation $[11,12]$. In this study, we mainly focus on the latter two steps. After sequence alignment and variant calling, we end up with a list of variants with their genomic coordinates and the variant alleles that differ from the reference sequence. Based on current knowledge of functional elements annotated in the human genome sequence, overlapping variants within the annotated features are found and the impact on RNA and protein sequence level is computed. Variant lists can be reduced further by applying functional impact prediction tools, such as Polyphen2 [13], SIFT [14], FoldX [15], and others [16]. Such tools are computationally intensive and require dedicated

(C) 2012 Sifrim et al.; licensee BioMed Central Ltd. This is an open access article distributed under the terms of the Creative Commons 
computing infrastructure to run variants in a highthroughput automated fashion. To tackle this problem, precomputed whole-genome predictions for popular tools are available in Ensembl and in dedicated databases [17]. Aggregated functional impact scores, such as CAROL [18], are also useful as these usually perform better in classification benchmarks for disease-causing mutations but are often not provided by current annotation tools.

These annotated variants can then further be interpreted by filtering based on either quality, functional, or genetic criteria. These filtered annotated data are then interpreted by either collapsing the data into the desired functional units, often genes, or performing formal statistical association analysis methods [19].

Several software tools have already been developed to tackle the aforementioned analysis steps. Some of these tools, such as ANNOVAR [20], TREAT [21], VarioWatch [22], SeqAnt [23], and Anntools [24], have been specifically designed to handle the annotation task and use diverse data sources in order to achieve this (Table 1). Other tools focus solely on the interpretation step and facilitate tasks such as easy filtering of data (VarSifter [25]), ranking based on putative pathogenicity (Var-MD [26]) or do more complex types of analysis by performing association analyses or looking at different underlying genetic disease models (VAAST [27]). A variety of tools try to be more comprehensive and provide a streamlined approach by tackling both steps (KGGSeq [28], SVA [29]).

Although current tools have matured significantly, they still possess several limitations. First there is the 'red queen' annotation problem. As functional information is constantly evolving, end users are forced to keep data sources and variant annotation up to date with every release of new information. Although some annotation tools make it easy to update annotation sources, this can still be a problem when trying to use previously acquired data sets in a related setting without repeating the complete annotation process. Secondly, interpretation is often done at the level of the individual sample, whereas cross-sample analysis is often left to the end user. This requires computational expertise when dealing with large data sets. Additionally different biological contexts might require a different combination of analysis techniques (that is, case/control studies versus familial or trio studies). Also, these analysis techniques are often phenotype-naive and disregard existing a priori biological knowledge of the particular disease or phenotype under study. Thirdly, when working in a collaborative setting, keeping track of individual files, annotation versions, and variants can prove difficult and easy sharing of variation, validation, sample, and experiment metadata is usually crucial. This is especially the case in the analysis of
Mendelian disorders where cases are often spread globally across research institutions.

\section{Aim}

To tackle these hurdles, we developed a framework, called Annotate-it, that provides experimentalists with a Swiss-knife approach for the interpretation of singlenucleotide variants, providing features such as automated annotation, prioritization of mutated genes, cross-sample querying and data management. We expand on existing comprehensive annotation and interpretation frameworks by integrating more annotation sources and different established state-of-the-art analysis techniques. We also integrate two different gene prioritization techniques: AGeneApart [30] and Endeavour [31]. AGeneApart mines MEDLINE abstracts to discover known genes or potentially new genes linked to the phenotype whereas Endeavour ranks genes based on similarity profiling compared to known disease genes. The latter method is more suitable for finding novel disease genes as it does not require a direct relationship between gene and disease [32]. These methods allow us to rank mutated genes taking the phenotype into account. Finally, because of its web-based interface, we allow for a collaborative workflow and the management of shared or public data, as well as a wide range of visualizations.

\section{Implementation}

\section{Annotation and filtering methodology}

Multiple annotation sources are supported, which can be classified into gene level and variant level information. Genes are annotated using Gene Ontology [33] terms, Online Mendelian Inheritance in Man (OMIM) and haploinsufficiency predictions [34]. We also provide pathway information from KEGG [35], BIOCARTA, and Reactome [36], as well as protein-protein interaction and protein complex data from STRING [37] and the Corum project [38]. Additionally, we provide statistical phenotypic associations with known chromosomal aberrations and phenotypic ontologies based on text-mining of recent MEDLINE releases [30]. Tissue-based expression information from the eGenetics/SANBI data set is also included to search for genes that are expressed in particular tissues of interest.

Information on the individual variants include PhastCons and Phylop scores (for primate, placental mammal and vertebrate multiple alignments) and presence in dbSNP, 1000 Genomes Project, and the 200 publicly available Danish exomes [39]. Also functional impact prediction scores from SIFT [14], Polyphen2 [13], LRT [40], and MutationTaster [41] are extracted from dbNSFP [17]. An aggregate score of SIFT and Polyphen2 is also computed with CAROL [18], which has been 
Table 1 Comparison of features of different tools for next-generation sequencing annotation and interpretation

\begin{tabular}{|c|c|c|c|c|c|c|c|c|c|c|c|}
\hline & ANNOVAR & TREAT & VAAST & VarSifter & Var-MD & KGGSeq & SVA & Anntools & VarioWatch & SeqAnt & $\begin{array}{l}\text { Annotate- } \\
\text { it }\end{array}$ \\
\hline Interface & $\begin{array}{l}\text { Command } \\
\text { line }\end{array}$ & $\begin{array}{l}\text { Command } \\
\text { line }\end{array}$ & & & & $\begin{array}{l}\text { Command } \\
\text { line }\end{array}$ & Graphical & $\begin{array}{l}\text { Python } \\
\text { script }\end{array}$ & $\begin{array}{l}\text { Command } \\
\text { line }\end{array}$ & & Graphical \\
\hline Command line & Web & Web & Web & & & & & & & & \\
\hline Indels & $x$ & $x$ & $x$ & $x$ & & $x$ & $x$ & $x$ & & $x$ & \\
\hline SNPS & $x$ & $x$ & $x$ & $x$ & $x$ & $x$ & $x$ & $x$ & $x$ & $x$ & $x$ \\
\hline miRNA annotation & $x$ & & & & & & & $x$ & & & $x$ \\
\hline $\begin{array}{l}\text { Regulatory region } \\
\text { annotation }\end{array}$ & $x$ & $x$ & & & & $x$ & & $x$ & & & \\
\hline $\begin{array}{l}\text { Custom } \\
\text { annotation }\end{array}$ & $x$ & & & & & & $x$ & $x$ & & & \\
\hline Gene annotation & $x$ & $x$ & & & & $x$ & $x$ & $x$ & $x$ & $x$ & $x$ \\
\hline $\begin{array}{l}\text { Conservation } \\
\text { scores }\end{array}$ & $x$ & $x$ & & & & $x$ & & & & $x$ & $x$ \\
\hline LRT & $x$ & & & & & $x$ & & & & & $x$ \\
\hline MutationTaster & $x$ & & & & & $x$ & & & & & $x$ \\
\hline Polyphen2 & $x$ & $x$ & & & & $x$ & & & & & $x$ \\
\hline SIFT & $x$ & $x$ & & & & $x$ & & & $x$ & & $x$ \\
\hline Aggregate scores & & & & & & $x$ & & & & & $x$ \\
\hline $\begin{array}{l}\text { Haploinsufficiency } \\
\text { prediction }\end{array}$ & & & & & & & & & & & $x$ \\
\hline OMIM & & $x$ & & & & & $x$ & & $x$ & & $x$ \\
\hline NHLBI Exomes & $x$ & & & & & & & & & & $x$ \\
\hline $\begin{array}{l}200 \text { Danish } \\
\text { Exomes }\end{array}$ & & & & & & & & & & & $x$ \\
\hline 1000 Genomes & $x$ & $x$ & & & & $x$ & $x$ & $x$ & $x$ & $x$ & $x$ \\
\hline $\mathrm{dbSNP}$ & $x$ & $x$ & & & & $x$ & $x$ & $x$ & $x$ & $x$ & $x$ \\
\hline Gene prioritization & & & & & & & & & & & $x$ \\
\hline Literature retrieval & & & & & & $x$ & & & $x$ & & $x$ \\
\hline Gene ontology & & & & & & & $x$ & & $x$ & & $x$ \\
\hline PPI/complexes & & & & & & $x$ & & & & & $x$ \\
\hline $\begin{array}{l}\text { Pathway } \\
\text { information }\end{array}$ & & $x$ & & & & $x$ & $x$ & & $x$ & & $x$ \\
\hline Gene expression & & $x$ & & & & & & & & & $x$ \\
\hline Statistical analysis & & & $x$ & & & & $x$ & & & & $x$ \\
\hline Filter & $x$ & $x$ & & $x$ & $x$ & $x$ & $x$ & & & & $x$ \\
\hline $\begin{array}{l}\text { Complex filtering } \\
\text { rules }\end{array}$ & & & & $x$ & & $x$ & $x$ & & & & $x$ \\
\hline $\begin{array}{l}\text { Cross-sample } \\
\text { querying }\end{array}$ & & & & $x$ & $x$ & $x$ & $x$ & & & & $x$ \\
\hline $\begin{array}{l}\text { Genetic } \\
\text { inheritance } \\
\text { models }\end{array}$ & & & $x$ & $x$ & $x$ & $x$ & $x$ & & & $x$ & $x$ \\
\hline Data sharing & & & & & & & & & & & $x$ \\
\hline Visualizations & & $x$ & & & & & $x$ & & $x$ & $x$ & $x$ \\
\hline
\end{tabular}

OMIM, Online Mendelian Inheritance in Man; PPI, protein-protein interaction.

demonstrated to perform better than individual scores at predicting deleteriousness of nonsynonymous variants.

Annotate-it has a flexible query system that allows answering complex cross-sample questions in an easy and quick way without any need for computational expertise. Using this interface, users can quickly check different inheritance hypotheses (for example, recessive or dominant models, or de novo occurrence of variants in parents-child trio analysis) depending on the available familial information. It also permits filtering based on consequence type, minimum and maximum coverage, minimum and maximum variant allele frequency, heterozygosity call, presence in dbSNP, 1000 Genomes Project, the NHLBI exome variant server and 200 Danish 
exomes, variants present in other samples, and conservation scores (Additional file 1). Annotate-it also allows screening a user-defined (or automatically generated) panel of genes of interest. Ultimately, these filtering steps will result in a list of genes (or exons) ordered by user-specified criteria (for example, present in given number of samples, number of unique alleles) (Additional file 2). Genes can then be further inspected by consulting all the available information that Annotate-it aggregates. This information contains associated phenotypes, automated literature retrieval, links to OMIM, region-based conservation plots, STRING or protein complex interaction partners and gene ontology terms (Additional file 3).

\section{Interpretation and statistical analysis}

In addition to the widely used collapsing method of disease-gene discovery, where variants across affected samples are collapsed at the gene level and counted, the user can also perform formal statistical analysis using the methodology proposed by Ionita-Laza for case/control studies [19]. This type of statistical methods has the added benefit of computing an approximate $P$-value for individual genes and of taking into account inherent background variation in genes due to factors such as gene size or hypermutable regions. However, they do require a significant amount of control samples in order to reach satisfying statistical power $(n>50)$. The analysis can be applied directly on the imported data without any reformatting or performing any command line actions.

The user can also provide a particular phenotype using Human Phenotype Ontology terms that can be used to derive genome-wide rankings produced by AGeneApart and Endeavour. These rankings are precomputed for all Human Phenotype Ontology terms for which at least five gene-phenotype associations are known, which can be used as a training set for the prioritization algorithm.

Furthermore the user can investigate mutation patterns of interaction partners of genes of interest. This is achieved by integrating the STRING and CORUM protein-protein interaction networks. This can be of particular interest when the cause of disease is expected to be oligogenic, such as diseases with some degree of phenotypic variability.

\section{Metadata and collaboration}

Additional metadata can be linked to samples and experiments. Samples are entities within experiments and can contain phenotypic information from different integrated ontologies (for example, European Paediatric Cardiac Code [42], London Dysmorphology Database [43], Human Phenotype Ontology [44]), gender, the date of sequencing, batch number, and other data. Metadata allow organizing and querying samples across multiple experiments. The experiments themselves can also store metadata, such as the software and parameters used for alignment and variant calling. Within an experiment, variants can be marked for validation, and validation information can be stored and reviewed. Promising candidate genes can be organized into gene lists when dealing with many collaborators or complex study setups. Gene lists can also be used as masking filters to either study gene specific gene panels or exclude sets of genes that are not of interest. An experiment-wide permission system with different roles (owner, editor, user) allows collaboration amongst different researchers and also allows the public sharing of data.

\section{Visual analytics}

Visual analytics is gaining ground for data exploration in the context of big data. The integrated interactive graphical filter (Figure 1) visualizes the different distributions of variants (for example, coverage, consequence types, conservation scores, variant allele frequency) and shows the impact of different filtering thresholds in real time, allowing selection of optimal thresholds. This is particularly useful in an exploratory phase to get a grasp on the characteristics of the data set and on how filter settings will affect these characteristics. Once optimal thresholds have been selected, they are easily applied in a subsequent discovery phase where the main goal is to select candidate genes of interest.

Calculating statistics for such large data sets in real-time is computationally taxing; therefore, Annotate-it's interactive filter can currently only be run on a subset of the variants (maximum 200,000 variants). Further research in the development of advanced data structures and browser infrastructure is needed to cope with the running time complexity of these approaches. As user interaction and feedback increases, we aim to optimize the informative value of such interconnected graphs and to improve the throughput of these visualization techniques.

\section{Technical specifications}

Annotate-it is programmed in the Ruby programming language and makes use of the Ruby on Rails framework [45] for its web interface. Currently, Annotate-it supports both hg18 (NCBI36) and hg19 (NCBI37) genomic builds. The annotation process leverages existing libraries, such as the Ruby Ensembl-API [46] and BioRuby [47], to enable programmatic access to locally run mirrors of the latest Ensembl [48] versions for any given genomic build. MySQL [49] was chosen to store variant information in a database. Nevertheless, Annotate-it's architecture is completely technology independent and therefore switching to different Rails-supported databases requires little effort. For large data sources containing billions of data points (such as base-wise conservation scores), we rely on Tokyo Cabinet [50] key-value stores for quick access. 


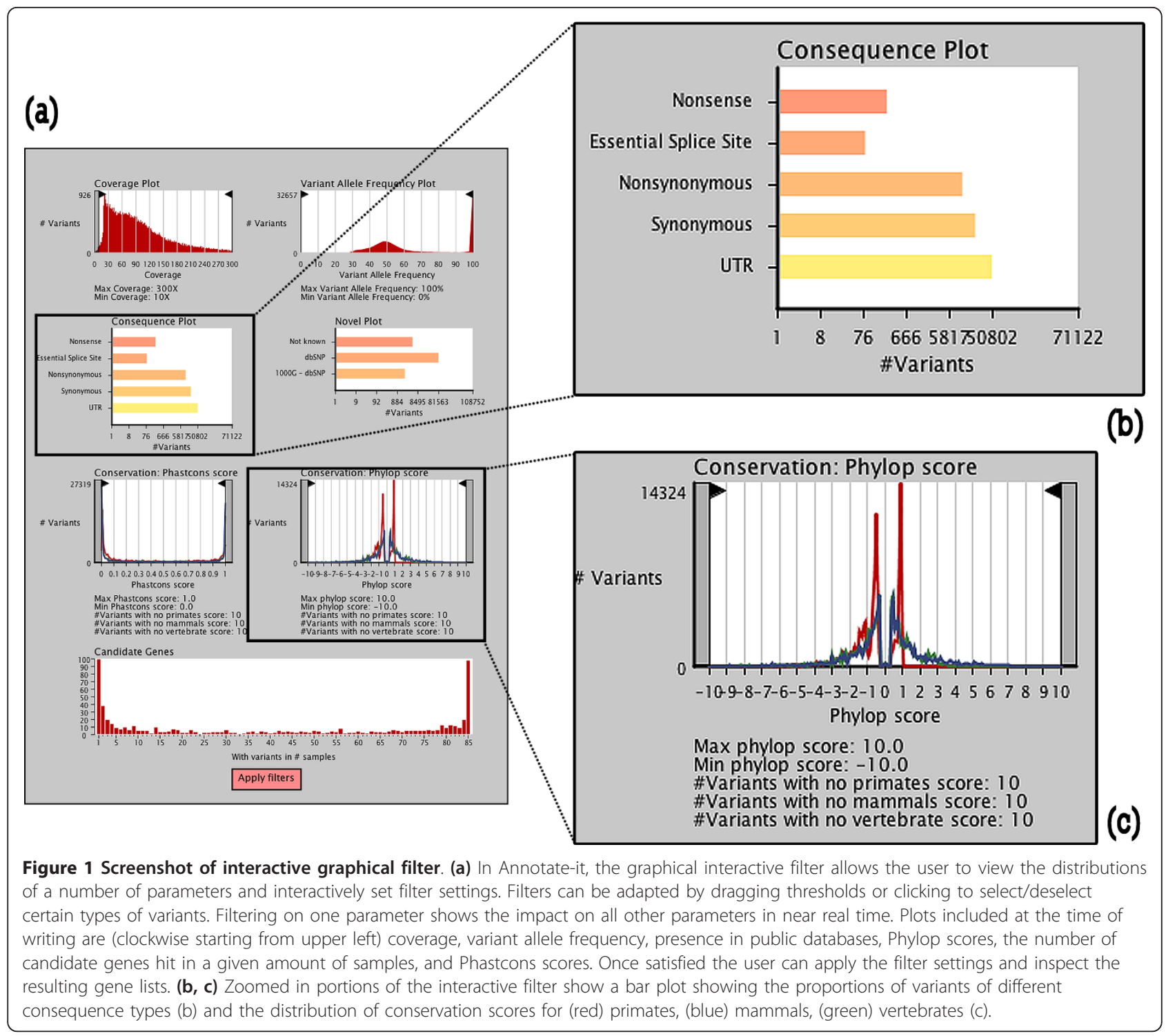

Interactive visualizations were implemented using Processing [33] and integrated into the web-interface as java applets.

Computationally intensive tasks (for example, complex predictions based on protein structure analysis) can be accomplished in a distributed manner by installing the Annotate-it client and the software one wishes to run on any computing node that has direct network access to the Annotate-it server. In this way, computing power can be scaled up on demand, either locally or by the use of cloudbased systems. Variant files can be uploaded and imported in the correct data structures at a pace of 500 variants per second using the current computing infrastructure. Previously unseen, and thus yet unannotated, variants are processed at a rate of approximately 85 variants per second. Computing infrastructure can be easily expanded to face increasing demand using elastic cloud technologies. This process is run completely at the server-side and requires no user interaction.

Input files of different formats are supported. So far, support includes Varscan [51], Atlas-SNP2 [52], Samtools [53] variant calling format, Genome Analysis Toolkit's VCF4 [54], Roche 454 GSMapper, and Illumina CASAVA output. Multiple files of different formats or sequencing technologies can be transparently combined for a single sample. Gene and variant lists can be exported to tabseparated value files based on the used filter-settings if needed.

\section{Generation of Miller syndrome semi-synthetic data sets}

We generated synthetic data sets of Miller syndrome by taking the following steps. We selected eight unrelated 
previously acquired exomes sequenced by Illumina GA2 and Illumina HiSEQ 2000. We aligned these exomes with BWA and called variants using the Genome Analysis Toolkit pipeline, both at default settings (Table 2). The sample identifiers of the variants were then randomly shuffled, maintaining the total number of variants, the number of variants per individual exome and the number of variants of each consequence type in the randomized data set (Figure 2). The eight synthetic exomes were then randomly assigned to either case or control groups so that each group contained four samples. Mutations found in the four published Miller syndrome cases were then added to each of the respective synthetic case samples. This synthetic case-control data set was then analyzed with Annotate-it using the following filter settings: minimum $10 \times$ coverage, minimum variant allele frequency of $20 \%$, only 'novel' variants, variants not found in the 1000 Genomes Pilot project, and variants not occurring in the control samples. Only nonsynonymous, nonsense or essential splice site variants were considered. Ranking of the candidate genes was done based on the number of case samples in which the gene was considered a hit and the Phylop conservation score based on the multiple alignment of placental mammals. We calculated rank statistics based on 1,000 randomizations following the previous steps.

\section{Results}

We validated the use of Annotate-it using three independent datasets: 1) a semi-synthetic Miller syndrome dataset, 2) a Schinzel-Giedion syndrome dataset and 3) a Nicolaides Baraitser syndrome dataset. In Miller syndrome as in Schinzel Giedion syndrome the causative gene was previously discovered and thus served to see if results could be reproduced using Annotate-it. Due to the synthetic nature of the Miller syndrome set we were able to statistically estimate the efficiency of commonly used filtering approaches in the discovery of Mendelian disease genes. In the Nicolaides Baraitser syndrome dataset the causative gene was previously unknown to us and

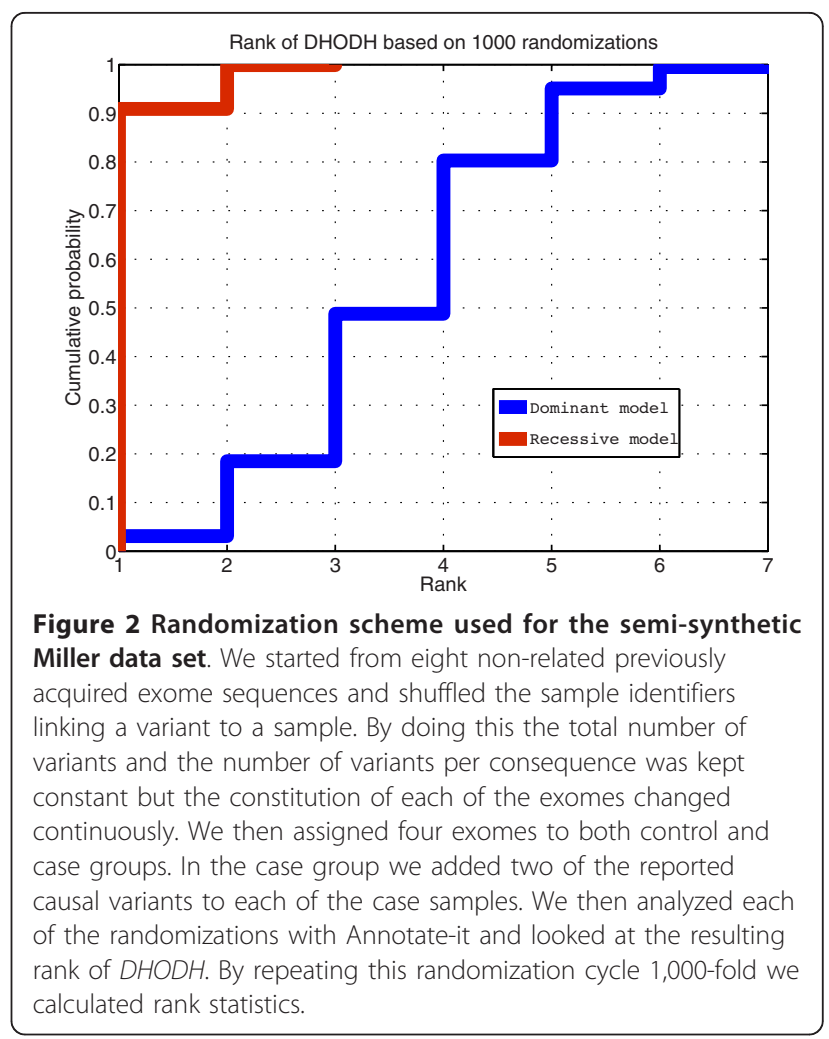

illustrates how additional phenotype-specific information can aid in the discovery of causative genes.

\section{Case study 1: semi-synthetic Miller syndrome data}

To evaluate the impact of random neutral variation on finding the causative gene, we generated a synthetic data set modeled on published cases of Miller syndrome [5], a rare recessive disease caused by mutations in $\mathrm{DHODH}$.

We considered two possible genetic models, which have been implemented in Annotate-it, when computing the rank of $D H O D H$ : the recessive model, meaning that a gene was only considered a hit in a sample if two different variants were found (assuming that a causal homozygous nonreference variant was highly unlikely), and the dominant

Table 2 Statistics of initial exomes used for randomizations

\begin{tabular}{lcccccccc}
\hline & \multicolumn{1}{c}{ Sample identifier } \\
\cline { 2 - 9 } Sample identifier & $\mathbf{1}$ & $\mathbf{2}$ & $\mathbf{3}$ & $\mathbf{4}$ & $\mathbf{5}$ & $\mathbf{6}$ & $\mathbf{7}$ & $\mathbf{8}$ \\
\hline Platform & GAll & GAll & GAll & GAll & HiSEQ & HiSEQ & HiSEQ & HiSEQ \\
Essential splice site mutations & 178 & 120 & 137 & 134 & 47 & 45 & 49 & 44 \\
Nonsense mutations & 99 & 88 & 95 & 108 & 101 & 60 & 68 & 68 \\
Nonsynonymous mutations & 8,864 & 7,468 & 8,039 & 8,246 & 7,925 & 6,715 & 6,487 & 6,560 \\
Synonymous mutations & 8,409 & 7,214 & 7,756 & 8,065 & 8,500 & 7,676 & 7,347 & 7,597 \\
UTR mutations & 3,839 & 3,216 & 3,080 & 3,264 & 4,264 & 1,443 & 1,421 & 1,367 \\
\hline
\end{tabular}

Eight previously acquired exomes were used to generate 8,000 synthetic exomes by randomization. The initial exomes were sequenced by either Illumina GA2 or Illumina HiSeq 2000, then aligned with BWA and variants were called using the GATK pipeline. 
model. Under the recessive model an average of $16( \pm 2)$ candidate genes with hits in the 4 patients $(41( \pm 4), 105$ $( \pm 6), 417( \pm 12)$ candidate genes with hits in at least 3,2 , or 1 patient, respectively) were found. Out of these candidate genes, $D H O D H$ ranked in the top two in $95 \%$ of the randomizations (Figure 3). Under the dominant model an average of $113( \pm 7)$ candidate genes with hits in the 4 patients (408 ( \pm 12$), 1132( \pm 15), 3417( \pm 15)$ candidate genes with hits in at least 3,2 , or 1 patient, respectively) were found. Out of these candidate genes, $D H O D H$ ranked in the top seven in $95 \%$ of the randomizations. These data show that, despite random neutral variation, simple filtering and sorting criteria can dramatically decrease the number of genes to be validated in the case of Mendelian disorders.

\section{Case study 2: Schinzel-Giedion syndrome}

In a study by Hoischen et al. [55] exome sequencing was used to identify causative mutations in Schinzel-Giedion syndrome. Because of the rarity of the disorder, the absence of gender bias, its mostly sporadic occurrence and the absence of cytogenetic imbalances, the disorder was believed to be caused by variants in a single or perhaps a few genes resulting in an autosomal dominant inheritance pattern. Exome sequencing of four patients, and validation in an additional four patients by Sanger sequencing, has identified de novo mutations in SETBP1 to be causative for the disease. These mutations were clustered in a highly conserved region of the gene and are found in an 11-bp stretch. In the previous study, mutations were filtered based on dbSNP build 130 for novel variants and only nonsynonymous (mutations changing the amino acid

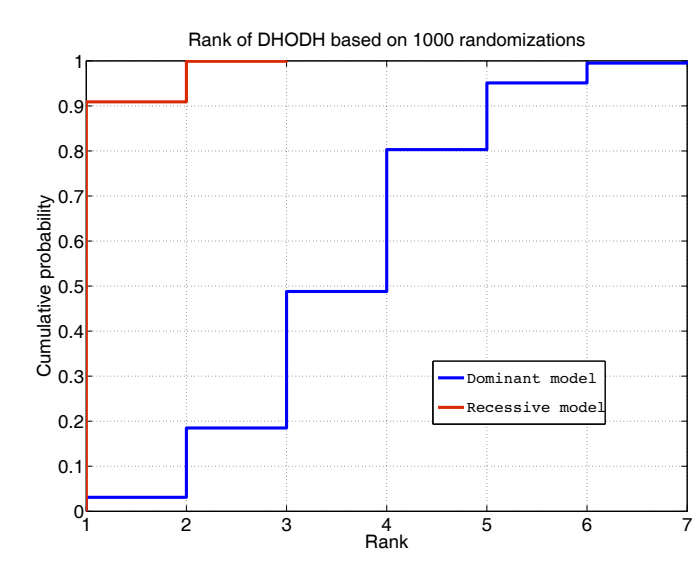

Figure 3 Rank statistics for DHODH based on 1,000 randomizations. The $x$-axis indicates the rank of $D H O D H$, the $y$-axis indicates the cumulative probability of observing a rank lower than that marked on the $x$-axis.(red) Under the recessive model $\mathrm{DHODH}$ ranked in the top two candidate genes in $95 \%$ of the randomizations. (blue). Under the dominant model $\mathrm{DHODH}$ ranked in the top seven in $95 \%$ of the randomizations. composition of the protein), nonsense (mutations resulting in either a gain or loss of a stop codon) or essential splice site variants (mutations located within two bases of the intron/exon boundary) were considered. Previously generated in-house data were used to filter out variants found in samples not related to the phenotype. This was done under the assumption that because of the rarity of the disorder, it was unlikely that these control samples would contain the same causal variant.

We reanalyzed the data using Annotate-it and applied default filters to validate the effectiveness of our methodology. We uploaded the variant files for annotation on the hg18/NCBI36 build of Annotate-it and filtered variants using two different settings: a cross-sample comparison at the gene level and a comparison at the exon level. The only major difference between both strategies is that instead of looking for candidate genes hit by variants across the patients, in the second approach we also look for specific exon hits across patients. For both strategies, we filtered out variants present in dbSNP (build 130) and the 1000 Genomes Project pilot studies (July 2010 release). We only selected variants with nonsense, nonsynonymous, and essential splice site consequences. We did not set any further filters, so as to recreate the scenario in which no further information was available to the researcher. Even though stricter filter settings would reduce the size of the resulting gene lists, we decided that manual inspection of all available information would allow us to select potential candidate genes without filtering a priori and potentially suffering false negative results that could lead to missing the implicated gene.

Both filtering approaches resulted in either a list of 11 candidate genes hit across the 4 patients (or 30, 91, 793 candidate genes in, respectively, at least 3, 2, or 1 patient) or 13 candidate exons across the 4 patients (or $38,81,840$ candidate exons in, respectively, at least 3, 2, or 1 patient). Sorting the gene list on number of samples implicated and maximum Phylop scores for placental mammals in the found variants, SETBP1 ranked at the top of the list as the prime candidate (Table 3). Further inspection of SETBP1 revealed the cluster of variants in a highly conserved region in the graphical view (Additional file 4), which might be indicative of gain-of-function mutations. Other genes that were hit across the four samples were also found but were deemed less likely because of either being hypervariable (for example, $C D C 27$ ) or having variants in regions of lower conservation. In the exon-based approach one of the SETBP1 exons was ranked in the top ten exons hit across the four patients. Other high ranking exons were deemed unlikely candidates because of the high number of variants found and were likely hypervariable regions or pseudogenes. This approach is theoretically more sensitive to distinguish causative exons, as neutral 
Table 3 Top ten candidate genes in the Schinzel-Giedion case study

\begin{tabular}{llccccc}
\hline Rank & Gene & $\begin{array}{c}\text { Number of } \\
\text { samples }\end{array}$ & $\begin{array}{c}\text { Number of unique } \\
\text { variants }\end{array}$ & $\begin{array}{c}\text { Total number of } \\
\text { variants }\end{array}$ & $\begin{array}{c}\text { Haploinsufficiency } \\
\text { prediction }\end{array}$ & $\begin{array}{c}\text { Maximum Phylop (placental } \\
\text { mammals) }\end{array}$ \\
\hline 1 & SETBP1 & 4 & 2 & 3 & 0.721 & - \\
2 & CDC27 & 4 & 5 & 13 & - & 3.455 \\
3 & CTBP2 & 4 & 3 & 8 & - & 2.805 \\
4 & PRB1 & 4 & 2 & 5 & - & 1.458 \\
5 & KIR2DL1 & 4 & 1 & 2 & - & 1.05 \\
6 & FLG & 4 & 5 & 6 & - & 1.034 \\
7 & OR11H1 & 4 & 0 & 1 & - & 0.856 \\
8 & KIR2DL3 & 4 & 4 & 6 & - & -0.326 \\
9 & CDCP2 & 4 & 0 & 1 & - & -0.365 \\
10 & NBPF12 & 4 & 0 & 2 & -0.411 \\
\hline
\end{tabular}

The data were analyzed using Annotate-it. Genes were considered a hit if they contained either nonsynonymous, nonsense or essential splice site variants that were not present in dbSNP or found in the 1000 Genomes Project. Genes were then sorted based on number of affected samples in which the gene was a candidate and conservation in placental mammals. Additionally, the total number of variants present across all samples and the number of variants unique to only one sample is given.

variation is less likely to be clustered in individual exons across samples, even though it remains susceptible to the previously mentioned regions of increased variability.

\section{Case study 3: Nicolaides-Baraitser syndrome}

Additionally we used Annotate-it to unravel the etiology of the previously unsolved Nicolaides-Baraitser syndrome (NBS) [56]. NBS is characterized by intellectual disability, sparse hair, distinctive facial characteristics, and distallimb anomalies. The extremely rare sporadic recurrence of the disease and the lack of bias towards any gender suggested the cause of the disease to likely be autosomal dominant de novo mutations. We collected DNA from four unrelated cases and performed whole-exome sequencing. We ran the analysis in Annotate-it using default filters and parameters (nonsense, splice site, and nonsynonymous mutations not present in any of the available population databases). This resulted in a list of 296 candidate genes sorted based on the number of samples having mutations in the given gene and the number of mutations pertaining to a single sample for that gene. The top ranked gene, $A C V R 2 A$, was the only candidate gene with variants present in the four samples containing the same variant. Recurring mutations are more likely to be population-specific polymorphisms, and thus not captured by aspecific population databases, than de novo variation, which is believed to randomly occur throughout the genome and therefore has a very low probability of mutating the same position four times in non-consanguineous individuals. The second best ranking gene, SMARCA2, contained unique heterozygous nonsynonymous mutations in three of the four samples. Further investigation of associated gene ontologies and associated phenotypes computed by AGeneApart revealed this gene to be involved in chromatin remodeling and facial abnormalities, making it an interesting candidate for validation. Sanger sequencing of the samples and their respective parents revealed the three variants to be $d e$ novo events. Additional resequencing of SMARCA2 in 44 individuals revealed heterozygous mutations in the same carboxy-terminal helicase domain in 36 patients, hereby validating SMARCA2 as the causative gene for Nicolaides-Baraitser syndrome. Later published studies discovered the etiology of the phenotypically related Coffin-Siris syndrome to be caused by similar mutations in SMARCB1 and ARID1B, which are direct interaction partners of SMARCA2 in the SWI/SNF chromatin remodeling complex $[57,58]$. Based on these findings we added functionality to Annotate-it to find causative mutations in protein complexes rather than in a strictly monogenic setting. By doing this we discovered that one of eight patients diagnosed with NBS for which we performed whole-exome sequencing carried a mutation in a gene identified in Coffin-Siris syndrome. Although the phenotype of NBS is strongly overlapping with that of Coffin-Siris syndrome, the latter differs by the presence of hypoplastic or absent fifth finger nails with or without hypoplasia of the terminal phalanges, the absence of swelling of the finger joints, short metacarpals and broad terminal phalanges and internal organ malformations are more common. Yet due to the large overlap of features, both syndromes are hard to classify [59], especially at a young age, which could explain the misclassification of this particular sample.

\section{Discussion \\ Context}

A multitude of publicly available computational tools exist in order to assist bioinformaticians and geneticists by facilitating the analysis of single nucleotide variants at the level of annotation, interpretation or both. Yet most tools focus only on particular aspects of the analysis pipeline (Table 1) 
and are thus only usable under certain experimental circumstances. Furthermore, all described tools suffer from several drawbacks, such as 'red queen' annotation problems, cross-sample analysis, phenotype-naive interpretation and general data management problems, such as data compatibility and data sharing [60].

\section{Annotate-it}

In this paper, we describe Annotate-it, a versatile framework for the analysis of multisample single nucleotide substitution data generated by next-generation sequencing. Annotation of samples is performed on the server side, eliminating the need for the installation of complex tools and annotation sources by the end-user and automatically keeping those annotations up to date.

The query and filtering interface enables the geneticist to quickly test different genetic hypotheses (recessive, dominant, de novo) across multiple samples and aggregates available information at the gene and variant level, facilitating the manual revision of candidate gene lists. We also provide a suite of more complex analysis techniques, such as aggregate functionality scores, phenotype-specific gene prioritization, and statistical methods for diseasegene finding in case/control studies without any need for data reformatting or computational expertise. By merging different state-of-the-art analysis techniques we aim to deliver a Swiss-knife type tool that can be used in many different experimental contexts. By unifying the interface and centralizing the data the user is able to perform multifaceted analyses without any need for additional data management or formatting and without requiring any computational expertise.

\section{Case studies}

To validate our approach we simulated an experiment with randomized exomes and published variants found in Miller syndrome patients. We computed ranking statistics showing that the causative gene ranks highly, even with a large amount of random neutral variation. This happens even when considering a relatively small set of patient exomes. In a second benchmark, we reanalyzed a published case study of the Schinzel-Giedion syndrome and identified the causative gene, SETBP1, as the topranked gene, using default parameters. Further inspection reinforced the previous finding that novel nonsynonymous variants across patients are clustered in a small 11-bp stretch. We applied the same approach in a previously unpublished study of NBS and found SMARCA2 as one of the top-ranking genes. This gene was then further validated to be the causative gene in this disease. Furthermore, we show in this case that gene prioritization methods can aid in the prioritization of mutated candidate genes by linking these genes to their associated phenotypes. Additionally, we show that in phenotypically overlapping disorders, leveraging protein-protein interaction data could prove useful in deciphering neighborhoods of mutated genes.

\section{Future perspectives}

Although the framework as presented here is fully able to support the discovery of causal genes of rare genetic disorders, our approach would benefit further from several critical features that we will investigate in future work. First, the confidentiality of human clinical data is a concern of many clinical geneticists and is still a topic of debate in most research centers. We aim to develop future releases of Annotate-it using a client-server architecture, so that sample and genotype information is dealt with on a locally installed client that automatically synchronizes genomic (but not clinical) data (that is, single nucleotide variant chromosome, position, and variant allele) with a server that holds the most up-to-date annotation information. A public client will be available for sharing data across research centers. Secondly, we designed Annotate-it with protein coding single-nucleotide substitutions in mind as proof-of-concept. We aim to further expand the scope of the methodology to encompass annotation of non-coding regions and other types of variation, such as insertions and deletions of varying lengths.

\section{Conclusions}

In this paper we describe the application of Annotate-it to study rare monogenic Mendelian disorders caused by rare, highly penetrant variants in the population. Because sequencing (as opposed to classical array-based genomewide association studies) detects rare (less than $0.01 \%$ of the general population), infrequent, and frequent variants (over 1\%), extensions of our strategy will be useful in resolving oligogenic diseases caused by a combination of infrequent variants of intermediate penetrance. Discovering the disease genes in such challenging cases will require good models of (1) the underlying population genetics (to avoid confounding effects from population structure), (2) the functional impact of variants on protein function and on regulation (to weed out passenger mutations), and (3) the biological pathways involved (to detect causative variants through their effect on pathways rather than individual genes). This is a primary research goal for the near future.

Current approaches to identifying causal variants by patient sequencing, including ours, can reliably interpret only the most straightforward subset of the variome (nonsense, missense, and splice-site variants). For example, synonymous variants, variants in regulatory, intronic, and intergenic regions, and variants in microRNAs remain highly challenging. Future experimental and in silico studies assessing the impact of such variants will allow us to develop computational strategies to bridge 
the gap in data interpretation between the exome and the complete genome. In particular, refinements in the mapping of regulatory regions and other functional elements through functional studies, evolutionary conservation, and in silico models will be essential to enable variant interpretation beyond the coding exome. Furthermore, the integration of large-scale structural variation will be crucial in a comprehensive study of the variome. Such integration will be challenging because structural variation inherently requires different types of data structure, visualization, and analysis methods. A high-level metaanalysis of variation at multiple scales should provide researchers with a complete picture of individual genomes. Making such complex, integrative analyses effective is a major challenge for the computational biology community.

Given the positive reactions from initial users, we believe that Annotate-it can play a significant role in the prioritization and identification of candidate genes in Mendelian disease. In the future, we aim at broadening its scope by increasing the annotation information available, the effectiveness and scope of our filtering analysis methods, and the robustness and ease of use of our web interface.

\section{Availability}

Annotate-it is freely available at [61]. Bug reports can be made and feedback accessed at [62].

\section{Requirements}

Annotate-it has been tested on the following browsers: Google Chrome (build 11.0.696.71 or later), Mozilla Firefox (version 3.6 or later), Safari (version 5.0 or later). Microsoft Internet Explorer is currently not supported.

\section{Additional material}

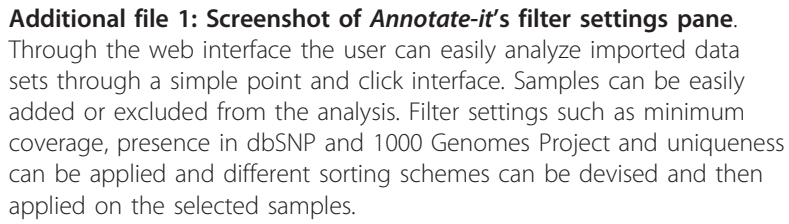

Additional file 2: Screenshot of Annotate-it's gene list view. After selecting samples to be analyzed and filtering and sorting criteria a resulting gene list is outputted. This gene list contains information on the amount of selected samples containing unfiltered variants and conservation scores. A tab with distribution plots is also provided (not shown here). Genes in this list are clickable and doing so returns more gene- and variant-specific information for that respective gene (Additional file 3).

Additional file 3: Screenshot of Annotate-it's gene details view Mutated genes can be further inspected to show additional information such as gene ontology, publications, associated pathways, associated phenotypes through gene prioritization, interaction partners (and their mutations) and involved protein complexes (and their mutations). Also detailed information on the mutations contained in that gene is given.
Additional file 4: Screenshot of Annotate-it's gene view visualization. This visualization shows the clustering of variants in an 11bp stretch of a single highly conserved exon of SETBP1 between position 40783844 and 40787303 . Conservation scores are given for the complete exon on the primate, mammal and vertebrate level. Nonsynonymous mutation are marked as light blue circles each belonging to one of the four patients (with sample identifiers 40816, 43664, 48062, 54126).

\section{Abbreviations}

bp: base pair; NBS: Nicolaides-Baraitser syndrome; OMIM: Online Mendelian Inheritance in Man; SNP: single nucleotide polymorphism.

\section{Acknowledgements}

The authors would like to thank Christian Gilissen en Joris Veltman for supplying the Schinzel-Giedieon data. Research supported by: Research Council KUL: ProMeta, GOA Ambiorics, GOA MaNet, CoE EF/05/007 SymBioSys en KUL PFV/10/016 SymBioSys, START 1, several PhD/postdoc \& fellow grants; Flemish Government: FWO: PhD/postdoc grants, projects, G.0318.05 (subfunctionalization), G.0553.06 (VitamineD), G.0302.07 (SVM/ Kernel), research communities (ICCOS, ANMMM, MLDM); G.0733.09 (3UTR); G.082409 (EGFR), IWT Grant No. IWT-SB/093289: PhD Grants, Silicos; SBOBioFrame, SBO-MoKa, TBM-IOTA3, FOD:Cancer plans, IBBT, Belgian Federal Science Policy Office: IUAP P6/25 (BioMaGNet, Bioinformatics and Modeling: from Genomes to Networks, 2007-2011); EU-RTD: ERNSI: European Research Network on System Identification; FP7-HEALTH CHeartED. BN is supported by KOLUMB fellowship from the Foundation for Polish Science.

\section{Author details}

${ }^{1} \mathrm{KU}$ Leuven, Department of Electrical Engineering-ESAT, SCD-SISTA, Kasteelpark Arenberg 10, B-3001, Leuven, Belgium. ${ }^{2}$ IBBT Future Health Department, Kasteelpark Arenberg 10, B-3001, Leuven, Belgium. ${ }^{3}$ KU Leuven, Centre for Human Genetics, University Hospital Gasthuisberg, Herestraat 49, 3000 Leuven, Belgium.

\section{Authors' contributions}

AS developed and maintained the system, prepared the first draft of the manuscript and reanalyzed the case study data; $A S, J K J V H, Y L, B N, L C T, R S$, $K D, J R V, Y M$ and JA contributed conceptually to the project. LCT precomputed all gene prioritizations; AS, RS, GAP and JA contributed to the visual interactive interface; $A S, J K J V H$ and BN tested the software. JRV and KD supervised the clinical genetic aspects of the project. YM and JA were the main supervisors of the project. All authors contributed equally to the final manuscript.

\section{Competing interests}

The authors declare that they have no competing interests.

Received: 31 August 2012 Revised: 14 September 2012

Accepted: 26 September 2012 Published: 26 September 2012

\section{References}

1. Altshuler D, Durbin RM, Abecasis GR, Bentley DR, Chakravarti A, Clark AG, Collins FS, De La Vega FM, Donnelly P, Egholm M, Flicek P, Gabriel SB, Gibbs RA, Knoppers BM, Lander ES, Lehrach H, Mardis ER, McVean GA, Nickerson DA, Peltonen L, Schafer AJ, Sherry ST, Wang J, Wilson R, Gibbs RA, Deiros D, Metzker M, Muzny D, Reid J, Wheeler D, et al: A map of human genome variation from population-scale sequencing. Nature 2010, 467:1061-1073.

2. Altshuler DM, Gibbs RA, Peltonen L, Dermitzakis E, Schaffner SF, Yu F, Bonnen PE, de Bakker PIW, Deloukas P, Gabriel SB, Gwilliam R, Hunt S, Inouye $M$, Jia X, Palotie A, Parkin M, Whittaker P, Chang K, Hawes A, Lewis LR, Ren Y, Wheeler D, Marie Muzny D, Barnes C, Darvishi K, Hurles M, Korn JM, Kristiansson K, Lee C, McCarroll SA, et al: Integrating common and rare genetic variation in diverse human populations. Nature 2010, 467:52-58.

3. Lupski JR, Reid JG, Gonzaga-Jauregui C, Rio Deiros D, Chen DCY, Nazareth L, Bainbridge $M$, Dinh $H$, Jing $C$, Wheeler DA, McGuire AL, Zhang F, 
Stankiewicz P, Halperin JJ, Yang C, Gehman C, Guo D, lrikat RK, Tom W, Fantin NJ, Muzny DM, Gibbs RA: Whole-genome sequencing in a patient with Charcot-Marie-Tooth neuropathy. N Engl J Med 2010, 362:1181-91.

4. Ng SB, Bigham AW, Buckingham KJ, Hannibal MC, McMillin MJ, Gildersleeve HI, Beck AE, Tabor HK, Cooper GM, Mefford HC, Lee C, Turner EH, Smith JD, Rieder MJ, Yoshiura K, Matsumoto N, Ohta T, Niikawa N, Nickerson DA, Bamshad MJ, Shendure J: Exome sequencing identifies MLL2 mutations as a cause of Kabuki syndrome. Nat Genet 2010, 42:790-793.

5. Ng SB, Buckingham K, Lee C, Bigham AW, Tabor HK, Dent KM, Huff CD, Shannon PT, Jabs EW, Nickerson DA, Shendure J, Bamshad MJ: Exome sequencing identifies the cause of a mendelian disorder. Nat Genet 2010, 42:30-35.

6. Ng SB, Turner EH, Robertson PD, Flygare SD, Bigham AW, Lee C, Shaffer T, Wong M, Bhattacharjee A, Eichler EE, Bamshad M, Nickerson DA, Shendure J: Targeted capture and massively parallel sequencing of 12 human exomes. Nature 2009, 461:272-276.

7. Postma AV, van Engelen $K$, van de Meerakker J, Rahman T, Probst $S$, Baars MJH, Bauer U, Pickardt T, Sperling SR, Berger F, Moorman AFM, Mulder BJM, Thierfelder L, Keavney B, Goodship J, Klaassen S: Mutations in the sarcomere gene MYH7 in Ebstein anomaly. Circ Cardiovasc Genet 2011, 4:43-50.

8. Tarpey PS, Smith R, Pleasance E, Whibley A, Edkins S, Hardy C, O'Meara S, Latimer C, Dicks E, Menzies A, Stephens P, Blow M, Greenman C, Xue Y, Tyler-Smith C, Thompson D, Gray K, Andrews J, Barthorpe S, Buck G, Cole J, Dunmore R, Jones D, Maddison M, Mironenko T, Turner R, Turrell K, Varian J, West $\mathrm{S}$, Widaa $\mathrm{S}$, et al: A systematic, large-scale resequencing screen of Xchromosome coding exons in mental retardation. Nat Genet 2009, 41:535-543.

9. Vissers LELM, de Ligt J, Gilissen C, Janssen I, Steehouwer M, de Vries P, van Lier B, Arts P, Wieskamp N, del Rosario M, van Bon BWM, Hoischen A, de Vries BBA, Brunner HG, Veltman JA: A de novo paradigm for mental retardation. Nat Genet 2010, 42:1109-1112.

10. Gilissen C, Arts HH, Hoischen A, Spruijt L, Mans DA, Arts P, van Lier B, Steehouwer M, van Reeuwijk J, Kant SG, Roepman R, Knoers NVAM, Veltman JA, Brunner HG: Exome sequencing identifies WDR35 variants involved in Sensenbrenner syndrome. Am J Hum Genet 2010, 87:418-423.

11. Magi A, Benelli M, Gozzini A, Girolami F, Torricelli F, Brandi ML: Bioinformatics for next generation sequencing data. Genes 2010, 1:294-307.

12. Flicek $P$, Birney E: Sense from sequence reads: methods for alignment and assembly. Nat Methods 2009, 6:56-S12.

13. Adzhubei IA, Schmidt S, Peshkin L, Ramensky VE, Gerasimova A, Bork P, Kondrashov AS, Sunyaev SR: A method and server for predicting damaging missense mutations. Nat Methods 2010, 7:248-249.

14. Ng P, Henikoff S: SIFT: Predicting amino acid changes that affect protein function. Nucleic Acids Res 2003, 31:3812.

15. Schymkowitz J, Borg J, Stricher F, Nys R, Rousseau F, Serrano L: The FoldX web server: an online force field. Nucleic Acids Res 2005, 33:W382-388.

16. Cline $M$, Karchin R: Using bioinformatics to predict the functional impact of SNVs. Bioinformatics (Oxford, England) 2010, 27:441.

17. Liu X, Jian X, Boerwinkle E: dbNSFP: a lightweight database of human non-synonymous SNPs and their functional predictions. Hum Mutat 2011, 32:894-899.

18. Lopes MC, Joyce C, Ritchie GRS, John SL, Cunningham F, Asimit J, Zeggini E: A combined functional annotation score for non-synonymous variants. Hum Hered 2012, 73:47-51.

19. Ionita-Laza I, Makarov V, Yoon S, Raby B, Buxbaum J, Nicolae DL, Lin X: Finding disease variants in Mendelian disorders by using sequence data: methods and applications. Am J Hum Genet 2011, 89:701-712.

20. Wang $\mathrm{K}, \mathrm{Li}$ M, Hakonarson H: ANNOVAR: functional annotation of genetic variants from high-throughput sequencing data. Nucleic Acids Res 2010, 38:e164.

21. Asmann YW, Middha S, Hossain A, Baheti S, Li Y, Chai H-S, Sun Z, Duffy PH, Hadad AA, Nair A, Liu X, Zhang Y, Klee EW, Kalari KR, Kocher J-PA: TREAT: a bioinformatics tool for variant annotations and visualizations in targeted and exome sequencing data. Bioinformatics (Oxford, England) 2012, 28:277-278.

22. Cheng Y-C, Hsiao F-C, Yeh E-C, Lin W-J, Tang C-YL, Tseng H-C, Wu H-T, Liu C-K, Chen C-C, Chen Y-T, Yao A: VarioWatch: providing large-scale and comprehensive annotations on human genomic variants in the next generation sequencing era. Nucleic Acids Res 2012, , 40 Web Server: W76-81.

23. Shetty AC, Athri P, Mondal K, Horner VL, Steinberg KM, Patel V, Caspary T, Cutler DJ, Zwick ME: SeqAnt: A web service to rapidly identify and annotate DNA sequence variations. BMC Bioinformatics 2010, 11:471.

24. Makarov V, O'Grady T, Cai G, Lihm J, Buxbaum JD, Yoon S: AnnTools: a comprehensive and versatile annotation toolkit for genomic variants. Bioinformatics (Oxford, England) 2012, 28:724-725.

25. Teer JK, Green ED, Mullikin JC, Biesecker LG: VarSifter: Visualizing and analyzing exome-scale sequence variation data on a desktop computer. Bioinformatics (Oxford, England) 2012, 28:599-600.

26. Sincan M, Simeonov D, Adams D, Markello TC, Pierson T, Toro C, Gahl WA Boerkoel CF: VAR-MD: A tool to analyze whole exome/genome variants in small human pedigrees with Mendelian inheritance. Hum Mutat 2012, 33:593-598.

27. Yandell M, Huff C, Hu H, Singleton M, Moore B, Xing J, Jorde LB, Reese MG: A probabilistic disease-gene finder for personal genomes. Genome Res 2011, 21:1529-1542.

28. Li M-X, Gui H-S, Kwan JSH, Bao S-Y, Sham PC: A comprehensive framework for prioritizing variants in exome sequencing studies of Mendelian diseases. Nucleic Acids Res 2012, 40:e53.

29. Ge D, Ruzzo EK, Shianna KV, He M, Pelak K, Heinzen EL, Need AC, Cirulli ET, Maia JM, Dickson SP, Zhu M, Singh A, Allen AS, Goldstein DB: SVA: software for annotating and visualizing sequenced human genomes. Bioinformatics (Oxford, England) 2011, 27:1998-2000.

30. Van Vooren S, Thienpont B, Menten B, Speleman F, De Moor B, Vermeesch J, Moreau Y: Mapping biomedical concepts onto the human genome by mining literature on chromosomal aberrations. Nucleic Acids Res 2007, 35:2533-2543.

31. Aerts $S$, Lambrechts $D$, Maity $S$, Van Loo P, Coessens B, De Smet F, Tranchevent L-C, De Moor B, Marynen P, Hassan B, Carmeliet P, Moreau Y: Gene prioritization through genomic data fusion. Nat Biotechnol 2006, 24:537-544.

32. Moreau Y, Tranchevent L-C: Computational tools for prioritizing candidate genes: boosting disease gene discovery. Nat Rev Genet 2012, 13:523-536.

33. Processing.. [http://www.processing.org/].

34. Huang N, Lee I, Marcotte EM, Hurles ME: Characterising and predicting haploinsufficiency in the human genome. PLoS Genet 2010, 6:e1001154.

35. Kanehisa M, Goto S, Sato Y, Furumichi M, Tanabe M: KEGG for integration and interpretation of large-scale molecular data sets. Nucleic Acids Res 2011, 40:D109-114.

36. Joshi-Tope G, Gillespie M, Vastrik I, D'Eustachio P, Schmidt E, de Bono B, Jassal B, Gopinath GR, Wu GR, Matthews L, Lewis S, Birney E, Stein L: Reactome: a knowledgebase of biological pathways. Nucleic Acids Res 2005, 33:D428-432.

37. Jensen L, Kuhn M, Stark M, Chaffron S, C: STRING 8 - a global view on proteins and their functional interactions in 630 organisms. Nucleic Acids Res 2009, 37:412-416.

38. Ruepp A, Waegele B, Lechner M, Brauner B, Dunger-Kaltenbach I, Fobo G, Frishman G, Montrone C, Mewes H-W: CORUM: the comprehensive resource of mammalian protein complexes - 2009. Nucleic Acids Res 2010, 38:D497-501.

39. Li Y, Vinckenbosch N, Tian G, Huerta-Sanchez E, Jiang T, Jiang H, Albrechtsen A, Andersen G, Cao H, Korneliussen T, Grarup N, Guo Y, Hellman I, Jin X, Li Q, Liu J, Liu X, Sparsø T, Tang M, Wu H, Wu R, Yu C, Zheng H, Astrup A, Bolund L, Holmkvist J, Jørgensen T, Kristiansen K, Schmitz O, Schwartz TW, Zhang X, Li R, Yang H, Wang J, Hansen T, Pedersen O, Nielsen R, Wang J: Resequencing of 200 human exomes identifies an excess of low-frequency nonsynonymous coding variants. Nat Genet 2010, 42:969-972.

40. Chun S, Fay JC: Identification of deleterious mutations within three human genomes. Genome Res 2009, 19:1553-1561.

41. Schwarz JM, Rödelsperger C, Schuelke M, Seelow D: MutationTaster evaluates disease-causing potential of sequence alterations. Nat Methods 2010, 7:575-576

42. Béland MJ, Franklin RCG, Jacobs JP, Tchervenkov Cl, Aiello VD, Colan SD, Gaynor JW, Krogmann ON, Kurosawa H, Maruszewski B, Stellin G, Weinberg PM: Update from the International Working Group for Mapping and Coding of Nomenclatures for Paediatric and Congenital Heart Disease. Cardiol Young 2004, 14:225-229.

43. Winter RM, Baraitser M: The London Dysmorphology Database. J Med Genet 1987, 24:509. 
44. Robinson PN, Köhler S, Bauer S, Seelow D, Horn D, Mundlos S: The Human Phenotype Ontology: a tool for annotating and analyzing human hereditary disease. Am J Hum Genet 2008, 83:610-615.

45. Ruby on Rails.. [http://rubyonrails.org/].

46. Strozzi F, Aerts J: A Ruby API to query the Ensembl database for genomic features. Bioinformatics (Oxford, England) 2011, 27:1013.

47. Goto N, Prins P, Nakao M, Bonnal R, Aerts J, Katayama T: BioRuby: Bioinformatics software for the Ruby programming language. Bioinformatics 2010, 26:2617.

48. Kersey PJ, Lawson D, Birney E, Derwent PS, Haimel M, Herrero J, Keenan S, Kerhornou A, Koscielny G, Kähäri A, Kinsella RJ, Kulesha E, Maheswari U, Megy K, Nuhn M, Proctor G, Staines D, Valentin F, Vilella AJ, Yates A: Ensembl Genomes: extending Ensembl across the taxonomic space. Nucleic Acids Res 2010, 38:D563-569.

49. MySQL.. [http://mysql.com/].

50. Tokyo Cabinet: a modern implementation of DBM.. [http://fallabs.com/ tokyocabinet/].

51. Koboldt DC, Chen K, Wylie T, Larson DE, McLellan MD, Mardis ER, Weinstock GM, Wilson RK, Ding L: VarScan: variant detection in massively parallel sequencing of individual and pooled samples. Bioinformatics (Oxford, England) 2009, 25:2283-2285.

52. Shen $Y$, Wan Z, Coarfa C, Drabek R, Chen L, Ostrowski EA, Liu Y, Weinstock GM, Wheeler DA, Gibbs RA, Yu F: A SNP discovery method to assess variant allele probability from next-generation resequencing data. Genome Res 2010, 20:273-280.

53. Li H, Handsaker B, Wysoker A, Fennell T, Ruan J, Homer N, Marth G, Abecasis G, Durbin R: The Sequence Alignment/Map format and SAMtools. Bioinformatics (Oxford, England) 2009, 25:2078-2079.

54. McKenna A, Hanna M, Banks E, Sivachenko A, Cibulskis K, Kernytsky A, Garimella K, Altshuler D, Gabriel S, Daly M, et al: The Genome Analysis Toolkit: A MapReduce framework for analyzing next-generation DNA sequencing data. Genome Res 2010, 20:1297.

55. Hoischen A, van Bon BWM, Gilissen C, Arts P, van Lier B, Steehouwer M, de Vries $P$, de Reuver R, Wieskamp N, Mortier G, Devriendt K, Amorim MZ, Revencu N, Kidd A, Barbosa M, Turner A, Smith J, Oley C, Henderson A, Hayes IM, Thompson EM, Brunner HG, de Vries BBA, Veltman JA: De novo mutations of SETBP1 cause Schinzel-Giedion syndrome. Nat Genet 2010, 42:483-485.

56. Van Houdt JKJ, Nowakowska BA, Sousa SB, van Schaik BDC, Seuntjens E, Avonce N, Sifrim A, Abdul-Rahman O, van den Boogaard M-JH, Bottani A, Castori M, Cormier-Daire V, Deardorff M, Filges I, Fryer A, Fryns J-P, Gana S, Garavelli L, Gillessen-Kaesbach G, Hall BD, Horn D, Huylebroeck D, Klapecki J, Krajewska-Walasek M, Kuechler A, Lines M, Maas S, Macdermot KD, McKee S, Magee A, et al: Heterozygous missense mutations in SMARCA2 cause Nicolaides-Baraitser syndrome. Nat Genet 2012, 44:445-449.

57. Santen GWE, Aten E, Sun Y, Almomani R, Gilissen C, Nielsen M, Kant SG, Snoeck IN, Peeters EAJ, Hilhorst-Hofstee Y, Wessels MW, den Hollander NS, Ruivenkamp CAL, van Ommen G-JB, Breuning MH, den Dunnen JT, van Haeringen A, Kriek M: Mutations in SWI/SNF chromatin remodeling complex gene ARID1B cause Coffin-Siris syndrome. Nat Genet 2012, 44:379-380.

58. Tsurusaki Y, Okamoto N, Ohashi H, Kosho T, Imai Y, Hibi-Ko Y, Kaname T, Naritomi K, Kawame H, Wakui K, Fukushima Y, Homma T, Kato M, Hiraki Y, Yamagata T, Yano S, Mizuno S, Sakazume S, Ishii T, Nagai T, Shiina M, Ogata K, Ohta T, Niikawa N, Miyatake S, Okada I, Mizuguchi T, Doi H, Saitsu H, Miyake N, Matsumoto N: Mutations affecting components of the SWI/SNF complex cause Coffin-Siris syndrome. Nat Genet 2012, 44:376-378

59. Sousa SB, Abdul-Rahman OA, Bottani A, Cormier-Daire V, Fryer A, GillessenKaesbach G, Horn D, Josifova D, Kuechler A, Lees M, MacDermot K, Magee A, Morice-Picard F, Rosser E, Sarkar A, Shannon N, Stolte-Dijkstra I, Verloes A, Wakeling E, Wilson L, Hennekam RCM: Nicolaides-Baraitser syndrome: Delineation of the phenotype. Am J Med Genet 2009, 149A:1628-1640.

60. Lyon G, Wang K: Identifying disease mutations in genomic medicine settings: current challenges and how to accelerate progress. Genome Med 2012, 4:58.

61. Annotate-it software.. [http://www.annotate-it.org].

62. Annotate-it feedback and bug reports.. [http://annotateit.userecho.com]. doi:10.1186/gm374

Cite this article as: Sifrim et al:: Annotate-it: a Swiss-knife approach to annotation, analysis and interpretation of single nucleotide variation in human disease. Genome Medicine 2012 4:73.

\section{Submit your next manuscript to BioMed Central and take full advantage of:}

- Convenient online submission

- Thorough peer review

- No space constraints or color figure charges

- Immediate publication on acceptance

- Inclusion in PubMed, CAS, Scopus and Google Scholar

- Research which is freely available for redistribution

Submit your manuscript at www.biomedcentral.com/submit
Biomed Central 